\title{
A randomised controlled trial comparing weight adjusted dose versus fixed dose prophylactic phenylephrine infusion on maintaining systolic blood pressure during caesarean section under spinal anaesthesia.
}

\author{
Lucy Mwaura, Vitalis Mung'ayi, Jimmie Kabugi, Samina Mir
}

\author{
Department of Anaesthesia, Aga Khan University, East Africa.
}

\begin{abstract}
Background: Spinal anaesthesia is the standard of care for elective caesarean delivery. It has advantages over general anaesthesia. However the sympathetic blockade induced by spinal anaesthesia results in an 80 percent incidence of hypotension without prophylactic management. Current evidence supports co-loading with intravenous fluids in conjunction with the use of vasopressors as the most effective way to prevent and treat the hypotension. Phenylephrine is the accepted vasopressor of choice in the parturient. A prophylactic phenylephrine infusion combined with a fluid co-load is proven to be an effective and safe method of maintaining maternal hemodynamic stability. While most published studies have assessed the effectiveness of a prophylactic phenylephrine fixed dose infusion, few studies have assessed the effect of a prophylactic phenylephrine weight adjusted dose infusion on maintaining maternal hemodynamic stability following spinal anesthesia for a cesarean delivery.

Objective: To compare the incidence of hypotension between women undergoing elective caesarean section under spinal anaesthesia, receiving prophylactic phenylephrine infusion at a fixed dose of 37.5 micrograms per minute versus a weight adjusted dose of 0.5 micrograms per kilogram per minute.

Methods: One hundred and eight patients scheduled for non-urgent caesarean section under spinal anaesthesia were randomized into 2 groups; control group and intervention group using a computer generated table of numbers.

Control group; Received prophylactic phenylephrine fixed dose infusion at 37.5 micrograms per minute.

Intervention group; Received prophylactic phenylephrine weight adjusted dose infusion at 0.5 micrograms per kilogram per minute

Results: The two groups had similar baseline characteristics in terms of ; Age, sex, weight and height. There was a $35.2 \%$ incidence of hypotension in the fixed dose group and an 18.6\% incidence of hypotension in the weight adjusted dose group. This difference was found to be of borderline statistical significance p-value 0.05 , and the difference in the incidence rates between the two groups was found to be statistically significant $\mathrm{p}=0.03$. The difference in the incidence of reactive hypertension and bradycardia between the two groups was not statistically significant: $\mathrm{p}$-value of 0.19 for reactive hypertension and $\mathrm{p}$-value of 0.42 for the incidence of bradycardia. There was also no statistically significant difference in the use of phenylephrine boluses, use of atropine, intravenous fluid used and the number of times the infusion was stopped.

Conclusion: Among this population, the incidence of hypotension was significantly less in the weight adjusted dose group than in the fixed dose group. There was no difference in the number of physician interventions required to keep the blood pressure within $20 \%$ of baseline, and no difference in the proportion of reactive hypertension or bradycardia between the two groups. Administering prophylactic phenylephrine infusion at a weight adjusted dose of 0.5 micrograms per kilogram per minute results in a lower incidence of hypotension compared to its administration at a fixed dose of 37.5 micrograms per minute.

Keywords: Weight adjusted dose, fixed dose prophylactic phenylephrine infusion, systolic blood pressure, caesarean section, spinal anaesthesia.
\end{abstract}

DOI: http://dx.doi.org/10.4314/ahs.v16i2.8

Cite as: Mwaura L, Mung'ayi V, Kabugi J, Mir S. A randomised controlled trial comparing weight adjusted dose versus fixed dose prophylactic phenylephrine infusion on maintaining systolic blood pressure during caesarean section under spinal anaesthesia. Afri Health Sci 2016;16(2): 399-411. bttp://dx.doi.org/10.4314/abs.v16i2.8

\author{
Corresponding author: \\ Vitalis Mung'ayi, \\ Department of Anaesthesia, \\ Aga Khan University, East Africa. \\ Phone: +254203662175 \\ Email: vitalis.mung'ayi@aku.edu/mungayi@gmail.com
}

\section{Introduction}

Spinal anaesthesia is the preferred mode of anaesthesia for elective caesarean delivery ${ }^{1,2}$. Benefits include avoiding the risks involved during general anaesthesia such as difficult intubation and aspiration of gastric contents ${ }^{3,4}$. Its use has been associated with reduced maternal mortality and better neonatal outcomes ${ }^{4}$. 
Hypotension is the most common complication of spinal anaesthesia ${ }^{5,6}$. It may be defined in terms of a systolic blood pressure of less than 90 or $100 \mathrm{mmHg}$ or in relative terms as a $20 \%$ decline of the systolic blood pressure from baseline ${ }^{7}$.

Consequences of untreated severe hypotension in the mother include: nausea and vomiting, cardiovascular collapse, unconsciousness with resulting pulmonary aspiration, and in extreme cases cardio respiratory arrest and to the baby: impaired placental perfusion leading to hypoxia, foetal acidosis and neurological injury ${ }^{8,9}$.

Measures taken to decrease the risk of hypotension include intravenous administration of fluids either crystalloids or colloids $5,10,11,12,13$, prevention of aortocaval compression by placing a wedge of $12 \mathrm{~cm}$ beneath the right buttock and use of vasopressors $\mathrm{s}^{5,7,8,14}$.

Ephedrine is an alpha and beta receptor agonist with both direct and indirect mechanism of action, but with a slow onset of action, making it difficult to titrate and use with an appropriate bolus dose $\mathrm{e}^{14}$.

Studies done have showed that ephedrine crosses the placenta and causes possible alterations in foetal physiology. It is also associated with a higher incidence of nausea and vomiting compared to phenylephrine $e^{4,15}$.

Phenylephrine is a short acting alpha agonist, it is a potent vasoconstrictor that counteracts the vasodilatation due to neuraxial anaesthesia and causes an increase in both systolic and diastolic blood pressures back to the baseline values ${ }^{7}$. It is widely used as studies done have showed better foetal acid base status with reduced incidence of foetal acidosis as well as lower episodes of maternal hypotension with the use of prophylactic phenylephrine regimens ${ }^{14,16}$. Various studies have found reduced incidence of hypotension and intra-operative nausea and vomiting when phenylephrine is administered as a prophylactic infusion at various doses compared with bolus administration and other methods, however no infusion regimen studied has been found to be more effective than others $^{17,18}$.

Hence, the best phenylephrine dose and mode of administration remains undetermined. Previously, studies have investigated its use as a prophylactic fixed dose infusion. There are no studies that have been done investigating how effective a prophylactic weight adjusted dose infu- sion would be, in terms of maintaining maternal systolic blood pressure within $20 \%$ of the baseline following spinal anaesthesia for caesarean section. Most drugs in anaesthesia as well as in medical practice, including vasopressors such as phenylephrine are administered at a weight adjusted dose as per medical guidelines/recommendations: thus supporting this study's investigation of a weight adjusted dose infusion.

This study was designed to assess how effective phenylephrine weight adjusted dose infusion regimen would be compared to a fixed dose infusion in maintaining maternal systolic blood pressure within $20 \%$ of baseline following spinal anaesthesia given for caesarean section.

Our study question was: does use of prophylactic phenylephrine weight adjusted dose infusion result in a lower incidence of hypotension following spinal anaesthesia given for caesarean delivery compared to its use as a prophylactic fixed dose infusion?

We hypothesised that a weight adjusted dose of prophylactic phenylephrine infusion results in a lower incidence of hypotension compared to a fixed dose infusion following spinal anaesthesia given for caesarean delivery. Our primary objective was to compare the incidence of hypotension between the two study groups. Our secondary objectives were to compare the incidence of reactive hypertension and bradycardia between the two groups and establish the number of physician interventions between the two study groups.

\section{Methods}

The study was performed following approval from the ethical and scientific review committee at the Aga Khan University Hospital, Nairobi. This was a prospective double blinded randomized controlled trial; the patient and physician administering anaesthesia and collecting data were blinded on the nature of the intervention made.

Since Nairobi is a cosmopolitan city, the patients served by this hospital cut across most racial groups present within the country. Patients were recruited from the outpatient pre-anaesthesia clinics and the maternity ward. The target population included all patients admitted for non-urgent caesarean section at the Aga Khan University Hospital, Nairobi. The sample population included 108 ASA I and II patients going to theatre for non-urgent 
caesarean section between October 2013 to January 2014. This comprised all eligible patients scheduled for nonurgent caesarean delivery who had given consent for the study.

All ASA I and II patients over 18 years scheduled for non-urgent caesarean delivery were included in this study. Reasons for exclusion from the study were:

1. Patient refusal to participate in the study

2. Patients with contraindications to neuraxial anaesthesia

a. Puncture site infection

b. Hypovolemic shock

c. Coagulopathy

d. Sepsis

e. Features of raised intracranial pressure

3. Patient involved in any other clinical studies

4. Women scheduled for emergency caesarean section

5. Type 1 Diabetes Mellitus

6. Hypertension

7. Severe cardiac disease graded as New York Heart Association Class (NYHA) III-IV

A sample size of 104 patients was sufficient to demonstrate a $17 \%$ difference in the proportion of hypotension between the two groups at $95 \%$ confidence level and a power of $80 \%$.

A study done by Allen et al showed the proportion of hypotension when a fixed dose of phenylephrine was used at $25 \mu \mathrm{g} / \mathrm{min}$ was $30 \%$ and $15 \%$ when a dose of $50 \mu \mathrm{g} / \mathrm{min}$ was used. ${ }^{17}$ By using phenylephrine at a dose of $37.5 \mu \mathrm{g} / \mathrm{min}$ we anticipated an incidence of $20 \%$. Our case series using prophylactic phenylephrine at a weight adjusted dose of $0.5 \mu \mathrm{g} / \mathrm{kg} / \mathrm{min}$ resulted in a much reduced incidence of hypotension at $3 \%$.

We therefore hypothesized that there would be a $17 \%$ less incidence of hypotension in the weight adjusted dose group as compared with the fixed dose infusion. Using this information and assuming a 5\% significance level and a power of $80 \%$, the required sample size was 52 patients in each group and a total of 104 patients. We recruited 108 patients to cater for any data entered wrongly, but all data was clearly entered so we analysed all patients.

Patients were recruited after having signed an informed consent, which clearly stated that it was a study being conducted and that their information would be kept con- fidential and may be published. Participant flow diagram is shown in figure 1.

No serious adverse event occurred to any patient for the entire duration of the study. The patients were free to withdraw from the study at any stage and still be accorded standard care.

Simple randomization was done using a computer program; the principal investigator generated a random sequence of numbers. Each of the random numbers was sequentially assigned to either;

Group 1; prophylactic phenylephrine fixed dose infusion at $37.5 \mu \mathrm{g} / \mathrm{min}$

Group 2; prophylactic phenylephrine weight adjusted dose infusion at $0.5 \mu \mathrm{g} / \mathrm{kg} / \mathrm{min}$.

The study drug was prepared by a physician not involved in any other aspect of the study in identical $20 \mathrm{mls}$ syringes labelled study drug. This physician held the code for randomization and group allocation. The drug was presented to the anaesthesiologist and the infusion rate was set at $20 \mathrm{mls}$ per hour for both groups. The presentation was similar and the only difference was the concentration of the drug in the syringe. The anaesthesiologist was also presented with a second $20 \mathrm{mls}$ syringe of phenylephrine $50 \mu \mathrm{g} / \mathrm{ml}$ for bolus phenylephrine administration in case systolic blood pressure fell below $20 \%$ of baseline.

On arrival to the operating theatres, standard monitoring was applied with automated non-invasive blood pressure measurement, electrocardiography and pulse-oximetry: with the objective of obtaining the baseline cardiovascular parameters. Baseline systolic blood pressure was obtained by taking three readings and calculating an average of the three readings.

After a local infiltration of $2 \mathrm{mls} 2 \%$ Lidocaine solution, a midline puncture with a 25 French gauge pencil point needle was performed at L3/L4 or L4/ L5 interspace, with the patient in the sitting or lateral decubitus position. After obtaining free flow of CSF, $2 \mathrm{mls}$ of bupivacaine and $10 \mu \mathrm{g}$ fentanyl were administered. Patients were then turned supine with a 15 degree left lateral tilt and the study drug together with the fluid co-load at 10mls/ $\mathrm{kg}$ were administered over 10 minutes. The study drug was run in an infusion pump at $20 \mathrm{mls}$ per hour as the concentration inside had already been predetermined and prepared. The sensory block level to both light touch and 
temperature was checked and a modified Bromage motor score recorded. Surgery was allowed to commence as soon as the sensory block had reached the desired level. NIBP was measured every 2 minutes till delivery of the baby.

Hypotension defined as a reduction in systolic blood pressure of more than $20 \%$ from baseline determined just before the administration of regional anaesthesia was treated with IV phenylephrine boluses of $50 \mu \mathrm{g}$. Reactive hypertension defined as a rise of systolic blood pressure above $20 \%$ of baseline was treated by stopping the infusion, and restarting it if blood pressure fell back to baseline. Bradycardia defined as heart rate less than 60bpm was treated by stopping the infusion if accompanied by reactive hypertension or with IV atropine $0.6 \mathrm{mg}$ if accompanied by hypotension. The presence of intraoperative nausea and vomiting was also noted and treated appropriately; Rescue antiemetic drugs (either IV ondansetron $4 \mathrm{mg}$ or granisetron $1 \mathrm{mg}$ ) were to be administered at the discretion of the anaesthesiologist. All the complications that occurred were noted and recorded by the anaesthesiologist.

The study drug infusion was discontinued after the delivery of the baby. APGAR score was recorded and othermanagement thereafter was at the discretion of the anaesthesiologist.

Intraoperative data was collected by the anaesthesiologist administering anaesthesia or research assistant both blinded to patients' group allocation using the data collection form. Upon collection, data was entered into the statistical software (SPSS version 15) on the same day in a coded form and saved, awaiting analysis. All data entered was verified by the principal investigator. There was no missing data and no patient required conversion to general anaesthesia.
Analysis was performed using STATA version 12 special edition. Descriptive statistics were used to compare patients' characteristics in terms of age, height, weight, baseline blood pressure and heart rates. Students' $T$ test was used to compare if the 2 sample sizes are statistically different. The Chi test was used to compare the proportions of hypotension between the two groups. The students' $T$ test was used to compare the differences between blood pressure and heart rates reduction between the two groups.

Observations (participants) were censored at the first event of interest i.e. hypotension or at the end of the observation period (end of surgery). Survival time analysis (Kaplan Meir) was used to analyse the time to hypotension. Log rank test was used to compare the rate of hypotension in the 2 groups.

The incidence rates (IR), and the incidence rate ratio (IRR) were reported alongside their corresponding 95\% CI. Kaplan-Meier survival curves were plotted. The test for differences in the survival distributions was conducted using log rank test.

The differences between the two groups in the total fluids given, total phenylephrine boluses and atropine used was compared using Mann-Whitney non parametric statistical test. All data was presented as mean +/- standard deviation unless otherwise specified. $\mathrm{P} \leq 0.05$ was considered statistically significant.

\section{Results}

One hundred and eight ASA 1 parturients scheduled to undergo non- urgent caesarean section were included in this study. Their baseline characteristics are shown in table 1. 
Table 1: Patients' baseline characteristics

\begin{tabular}{|l|l|l|l|}
\hline & $\begin{array}{l}\text { Fixed dose group } \\
\mathrm{n}=54 \\
\text { Mean SD }\end{array}$ & $\begin{array}{l}\text { Weight adjusted dose group } \\
\mathrm{n}=54 \\
\text { Mean SD }\end{array}$ & P-Value \\
\hline $\begin{array}{l}\text { Age in } \\
\text { years }\end{array}$ & 32.84 .8 & 33.53 .6 & 0.4 \\
\hline Weight $(\mathrm{kg})$ & 79.211 .7 & 82.910 .3 & 0.09 \\
\hline Height $(\mathrm{cm})$ & 162.51 .01 & 162.11 .09 & 0.2 \\
\hline
\end{tabular}

Data are mean + SD, T-test used to analyse normal distributed variables and Mann-Whitney U test for skewed data.

Table 2 shows the sensory block achieved before the start of surgery for the two study groups.

Table 2: sensory block level before start of surgery

\begin{tabular}{|l|l|l|}
\hline Sensory level & $\begin{array}{l}\text { Fixed dose group } \\
\mathrm{n} \%\end{array}$ & $\begin{array}{l}\text { Weight adjusted dose group } \\
\mathrm{n} \%\end{array}$ \\
\hline T4 & $11 \%$ & $20 \%$ \\
\hline T6 & $79 \%$ & $66 \%$ \\
\hline T8 & $10 \%$ & $14 \%$ \\
\hline
\end{tabular}

Table 3 shows the primary outcome as the proportion of hypotension in the two groups.

Table 3: Proportion of hypotension between the two groups

\begin{tabular}{|c|c|c|c|}
\hline & HYPOTENSION & & \\
\hline & $\mathrm{No}(\mathrm{n} \%$ & Yes(n\%) & Total(n $\%)$ \\
\hline Control fixed dose infusion & $3564.8 \%)$ & $19(35.2 \%$ & $54(100 \%)$ \\
\hline Intervention weight adjusted dose infusion & $44(81.4 \%)$ & $10(18.6 \%$ & $54(100 \%)$ \\
\hline TOTAL & $79(73.2 \%)$ & $29(26.8 \%)$ & $108(100 \%)$ \\
\hline P- Value & & & \\
\hline
\end{tabular}


Table 4 shows the incidence rate of hypotension during the period of study for the two study groups.

Table 4: Incidence rate of hypotension between the two groups

\begin{tabular}{|c|c|c|c|c|}
\hline & $\begin{array}{l}\text { Person time } \\
\text { minutes }\end{array}$ & $\begin{array}{l}\text { Hypotensive } \\
\text { patients(n) }\end{array}$ & $\begin{array}{l}\text { Rate } \\
\text { per } 100 \text { person } \\
\text { minutes }\end{array}$ & $\begin{array}{l}95 \% \text { confidence } \\
\text { intervals }\end{array}$ \\
\hline Fixed dose group & 636 & 19 & 3 & $1.9-4.7$ \\
\hline $\begin{array}{l}\text { Weight adjusted } \\
\text { dose group }\end{array}$ & 764 & 10 & 1.3 & $0.7-2.4$ \\
\hline Total & 1400 & 29 & 2.1 & $1.4-3$ \\
\hline Rate Ratio & & & 2.3 & \\
\hline Hazard Ratio & 0.43 & & & \\
\hline P-value & 0.035 & & & \\
\hline
\end{tabular}

The results on presence of bradycardia, reactive hyper- the control (fixed dose group) and the intervention tension and number of physician interventions between (weight adjusted group) are shown in tables 5, 6 and 7 respectively.

Table 5: Difference in the rates of reactive hypertension between the fixed dose group and the weight adjusted dose group.

\begin{tabular}{|l|l|l|l|l|}
\hline Group & $\begin{array}{l}\text { Proportion } \\
\mathrm{N} \%\end{array}$ & $\begin{array}{l}\text { Person } \\
\text { time }\end{array}$ & Rate & $95 \%$ Confidence Interval \\
\hline Fixed dose group & $1834 \%$ & 636 & 1.6 & $0.8-2.9$ \\
\hline $\begin{array}{l}\text { Weight adjusted dose } \\
\text { group }\end{array}$ & $1122 \%$ & 730 & 0.9 & $0.4-1.9$ \\
\hline \multicolumn{2}{|l|}{} \\
\hline
\end{tabular}

Table 6: Test for difference in the proportion of bradycardia between the control and the intervention arms.

\begin{tabular}{|l|l|l|l|}
\hline Group & Proportion & Incidence Rate & $95 \%$ Confidence Interval \\
\hline Fixed dose group & $41.5 \%$ & 4.2 & $2.7-6.2$ \\
\hline $\begin{array}{l}\text { Weight adjusted dose } \\
\text { group }\end{array}$ & $34.0 \%$ & 3.1 & $1.9-4.8$ \\
\hline $\mathrm{P}=0.349$ & \multicolumn{3}{|l|}{} \\
\hline
\end{tabular}


Table 7: Physician interventions.

\begin{tabular}{|l|l|l|l|}
\hline & $\begin{array}{l}\text { Control: Fixed dose } \\
\text { Percentage/Mean }\end{array}$ & $\begin{array}{l}\text { Intervention: weight adjusted dose } \\
\text { Percentage/Mean }\end{array}$ & P- Value \\
\hline Infusion stopped & $8(15 \%)$ & $5(9 \%)$ & 0.374 \\
\hline Phenylephrine bolus & $17(31 \%)$ & $10(19 \%)$ & 0.119 \\
\hline Atropine given & $7(13 \%)$ & $6(11 \%)$ & 0.73 \\
\hline Amount of Fluids used & $746 \mathrm{mls}$ & $712 \mathrm{mls}$ & 0.15 \\
\hline
\end{tabular}

The time from induction of spinal anaesthesia to delivery was similar in both groups (range from 15-40 minutes) as was the sensory level by cold touch before the start of surgery as shown in table 2 .

There was $35.2 \%$ incidence of at least one episode of hypotension in the control group (Fixed dose group) and $18.6 \%$ in the intervention group (weight adjusted group). This difference in the incidence of hypotension between the two groups was found to be of borderline statistical significance ( $\mathrm{p}$ value of 0.051 ) as shown in table 3 .

The overall total person time minutes to hypotension was 1400 minutes, with 636 minutes for the control arm and 764 in the intervention arm. The incidence rate of hypotension in the fixed dose group was 3 per 100 person minutes $95 \%$ CI 1.9- 4.7 compared to 1.3 per 100 person minutes 95\% CI: 0.7- 2.4 in the weight adjusted dose group. The rate ratio was 2.3 per 100 person minute the patients in the control group had twice the risk of developing hypotension compared to those in the intervention group and a hazard ratio of 0.43 , meaning that patients in the intervention arm had 57\% chance of protection from hypotension.
The test for differences in the two incidence rates the test of whether the IRR $=1$ against that it is different from 1 was statistically significant, $\mathrm{P}=0.035$. This was a coincidental finding however; as our study was not powered to detect this difference.

Based on the lower proportion of hypotension in the weight adjusted dose group compared to the fixed dose group table 3 and the much lower incidence rate of hypotension as illustrated in table 4, there is good evidence for the alternate hypothesis that a weight adjusted infusion results in a lower incidence of hypotension compared to a fixed dose infusion.

Figure 2 presents the survival distribution by the treatment group when the event of interest is hypotension. The Log rank test is not significant implying that the two survival distributions are not different, $\mathrm{P}$ value of 0.06 . This may be due to the closeness of the curves in the first 4 minutes. The curves however, are far from each other after 10 minutes. The intervention arm had a higher survival probability compared to the control arm, implying that the intervention arm had a reduced chance of developing hypotension after 10 minutes compared to the control. 


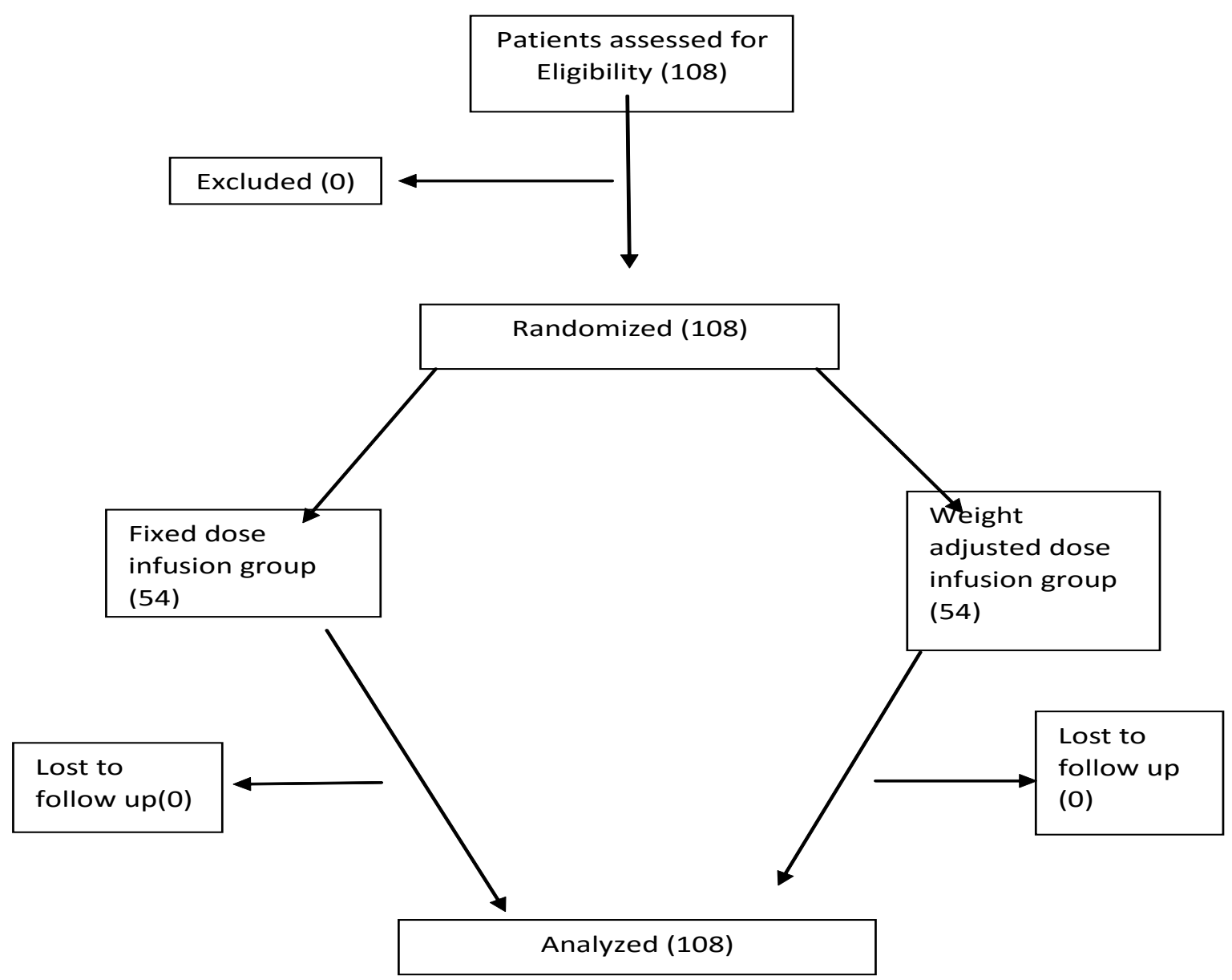

The rate of reactive hypertension was calculated for the episodes that occurred over the total person time of observation. The control arm had an incidence rate of 1.6 95\% CI: 0.8- 2.9 per 100 person minutes while the intervention arm had an incidence rate of hypertension of 0.9 95\% CI: 0.4- 1.9 per 100 person minutes. The intervention arm had a reduced risk of hypertension but the test for differences in the two incidence rates was not statistically significant, $\mathrm{P}=0.19$.

The fixed dose arm had an incidence rate of bradycardia of $4.295 \%$ CI: $2.7,6.2$ per 100 person minutes while the weight adjusted dose arm had an incidence rate of bradycardia of $3.195 \%$ CI: 1.9, 4.8 per 100 person minutes giving an incidence rate ratio IRR of $0.7495 \%$ CI: 0.0.37, 1.45. This implies that the weight adjusted dose arm had a reduced risk of bradycardia but the test for differences in the two incidence rates i.e. the test of whether the $I R R=1$ against that it is different from 1 was not statistically significant, $\mathrm{P}=0.349$.

Phenylephrine infusion was stopped in $13(12 \%)$ of all the patients, with $8(15 \%)$ and 5(9\%) in the control and intervention arms respectively. The Chi Square test for difference in proportions between the two arms Control: 8 (15\%); Intervention: 5 (9\%) showed no evidence of association between the control and the intervention arms, Chi Square value $=0.789$ and $\mathrm{P}=0.374$. 


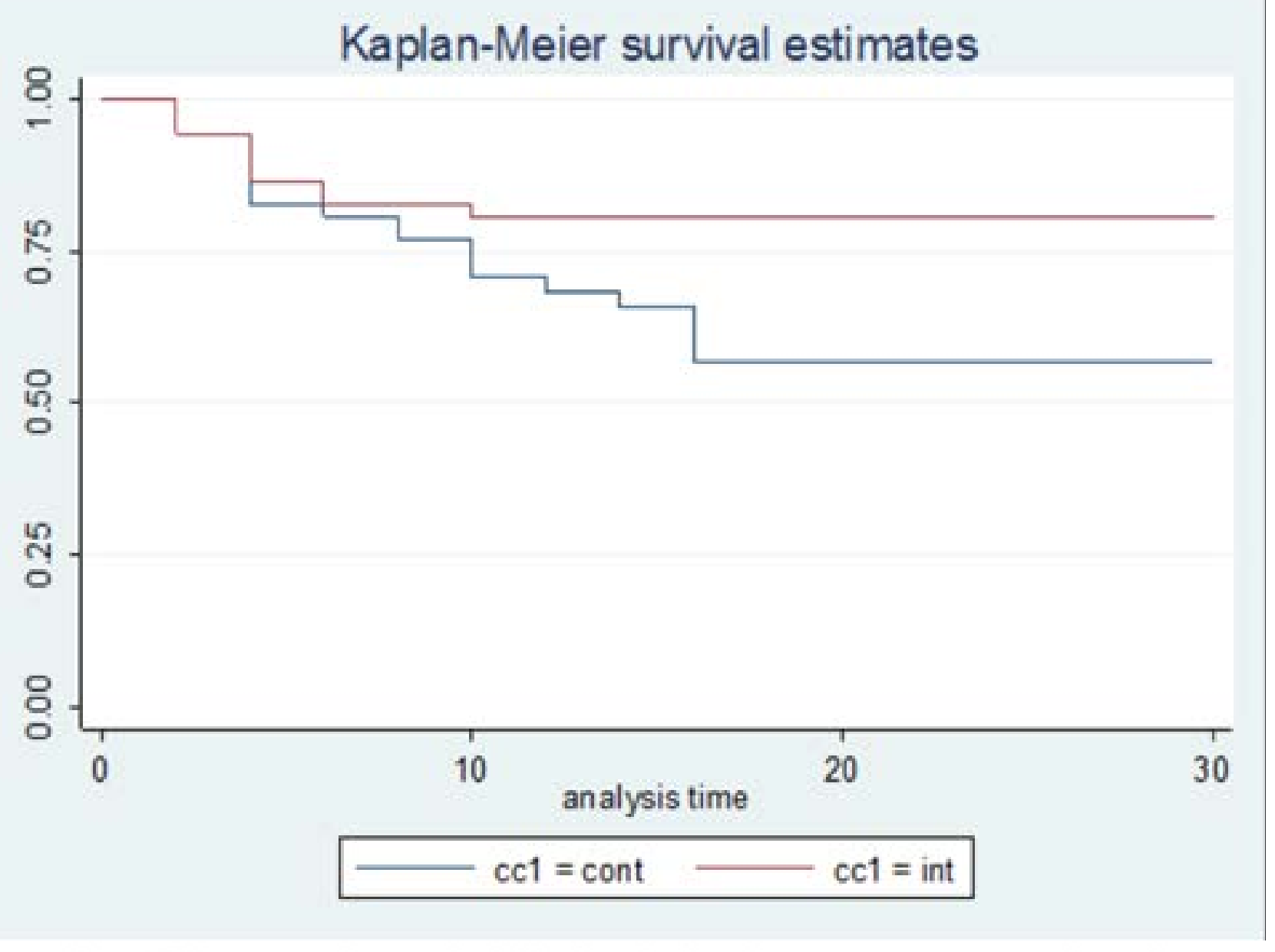

Figure 2: Presents the survival distribution by the treatment group when the event of interest is hypotension.

Rescue Phenylephrine bolus was given to $27(25 \%)$ participants. Of this number, 17(31\%) were from the control arm while $10(19 \%)$ were from the intervention arm. There was no evidence of any association between bolus and treatment, Chi Square value $=2.435, \mathrm{P}=0.119$.

Similarly, atropine was given to $13(12 \%)$ of the participants. The proportions of those who received atropine in the control and intervention arms were 6(11\%) and $7(13 \% 0$ respectively. The Chi Square test for association showed no evidence of association between the treatment and atropine, Chi Square value $=0.111$ and $P=0.739$. There was no significant difference in the amount of intravenous fluids Ringer's Lactate used between the two groups $\mathrm{p}=0.15$

\section{Discussion}

To the best of our knowledge, this is the first study ever investigating the use of prophylactic phenylephrine infu- sion at a weight adjusted dose in the prevention of maternal hypotension following spinal anaesthesia. It is also among the first studies investigating use of prophylactic phenylephrine infusion to prevent maternal hypotension in our patient population.

The principal finding of this study was that using a weight adjusted dose of phenylephrine infusion resulted in a lower proportion of hypotension compared to the use of a fixed dose infusion, this difference was of borderline statistical significance. Prophylactic weight adjusted dose infusion was also found to significantly reduce the incidence rate of hypotension compared to when a fixed dose infusion at $37.5 \mu \mathrm{g} / \mathrm{min}$ was used Table 4.

This study was however not powered to detect a difference in the incidence rates. The lower rate of hypotension in the weight adjusted dose group might be ex- 
plained by the pharmacokinetics of the drug, different doses of drugs result in different plasma concentration of the drug producing a range of therapeutic and some undesirable responses. Following administration of a drug, its plasma concentration rises to a maximum depending on; its volume of distribution and the dose that has been administered ${ }^{19}$. Using a standard or a fixed dose for all patients fails to account for the wide variation in volumes of distribution ${ }^{20}$. When a drug is dosed based on the patients' weight the assumption made is that the drug pharmacokinetic parameters increase as the body size increases ${ }^{20}$.This contrasts to using a fixed dose for all patients whereby the assumption is that the pharmacokinetic parameters remain constant despite changes in the body size ${ }^{20}$.

Phenylephrine is a synthetic, selective, sympathomimetic agent alpha 1 agonist. It is usually administered intravenously, it has potent vasoconstrictor properties that occur almost immediately but only last for 15-20 minutes hence it is necessary to administer it as an infusion ${ }^{21}$. It is metabolized in the liver and excreted by the kidneys, does not bind to plasma proteins and distributes widely throughout the extracellular fluid. Hence administration of phenylephrine in patients with varying weights suggests that weight adjusted phenylephrine dosing may be an important consideration. In our study, we used the lower limit of recommended weight adjusted dose of phenylephrine at $0.5 \mu \mathrm{g} / \mathrm{kg} / \mathrm{min}$, the reason for this was to prevent accumulation and toxicity that may occur in obese patients. In previous studies done on prophylactic phenylephrine infusions the mean weight of the patients studied was 65-70 kegs.

This contrasts to our study whereby the mean weight was $85 \mathrm{kgs}$, with a range of $60 \mathrm{kgs}-110 \mathrm{kgs}$. This might explain why a higher incidence of hypotension was observed in our patients when the recommended fixed dose regimen are used, 35.5\% versus 30\% reported by Allen et $\mathrm{al}^{17}$. James $\mathrm{T}$ Miller et al conducted a retrospective cohort study aimed at determining if the effects of vasopressin on blood pressure and other vasopressor requirement is related to body weight ${ }^{22}$. They demonstrated that the efficacy of vasopressin reflected by a decrease in other vasopressor requirement is significantly correlated to its dose when adjusted for body weight ${ }^{22}$. Despite the fact that this is a different patient population and a different drug, and that this was a retrospective study these two drugs are both vasopressors and the findings of this study support weight adjusting the dose of vasopressors to improve their efficacy.

The correct dosing approach can only be known if drug pharmacokinetics are tested across a wide range of weights: in our study the range was between 60-110kgs. Another assumption made with weight based dosing is that; there is a linear relationship between the clearance of a drug and the bodyweight, and that the clearance of a drug increases as the body weight increases ${ }^{20}$.The findings in this trial suggests that in an effort to maintain maternal systolic blood pressure as close to the baseline as possible using weight adjusted dosing, prophylactic phenylephrine infusion might offer the advantage of reducing the incidence rate of hypotension compared to using the infusion as a fixed dose for all patients regardless of their weight. This could be explained by the fact that by weight adjusting the dose, we avoid under dosing the drug in some patients and increased toxicity in other patients.

In our study, we defined hypotension as a $20 \%$ decrease in the systolic blood pressure from the baseline. Based on a case series of prophylactic phenylephrine infusion at $0.5 \mu \mathrm{g} / \mathrm{kg} / \mathrm{min}$, we had postulated that only $3 \%$ of patients in the weight adjusted group would develop hypotension for the entire duration of the study. The discrepancy between the postulated proportion in the intervention group and the results of the study was probably due to a few methodological flaws in the anecdotal case series: no protocol was followed in the administration of fluids and the sample size was also very small. Furthermore there is a paucity of published well designed studies that have used weight adjusted prophylactic phenylephrine infusion. The findings of this study are in keeping with our hypothesis; the proportion of hypotension was lower in the intervention arm compared to that in the control arm Table 3.

The findings in the control group correspond closely to the published proportion of hypotension of $23 \%$ by

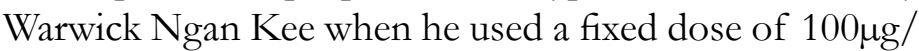
$\min ^{16}$ and that by Terrence et al who found a proportion of $30 \%$ when a fixed dose infusion at $25 \mu \mathrm{g} / \mathrm{min}$ was used. ${ }^{17}$ 
Reactive hypertension and bradycardia occur frequently and are a major concern with the use of prophylactic phenylephrine infusions. Although the differences in the incidence rate ratio was not statistically significant, the use of a weight adjusted infusion appeared to reduce the risk of reactive hypertension, Table 5 . This can again be explained by the fact that by using the weight adjusted dose especially in patients at the lower weight range we avoided overexposing patients to a higher dose than necessary that would have resulted in reactive hypertension and bradycardia. The proportions of reactive hypertension in this study closely compare to those reported by Allen et al, whereby $25 \%$ of patients developed reactive hypertension when a low dose infusion of $25 \mu \mathrm{g} / \mathrm{min}$ was used and $40 \%$ when a dose of $50 \mu \mathrm{g} / \mathrm{min}$ was used ${ }^{17}$. The proportion in the weight adjusted group was actually much lower than that seen when the low dose infusion was used at $25 \mu \mathrm{g} / \mathrm{min}$ in an attempt to reduce the proportion of reactive hypertension.

Phenylephrine infusion is associated with an increase in systemic vascular resistance and subsequently causes an increase in the blood pressure and a baroreceptor mediated bradycardia. In an editorial Robert A Dyer suggested use of phenylephrine at low infusion rates to reduce hypotension and avoid reactive hypertension and bradycardia associated with the use of phenylephrine infu$\operatorname{sion}^{23}$. The proportion of bradycardia shown in Table 6 was much higher than the proportion observed by Allen et al of $15 \%$ when a low dose of $25 \mu \mathrm{g} / \mathrm{min}$ was used. The reason for this is that, in their study bradycardia was defined as a heart rate of less than 50 beats/min unlike in our study where we defined it as a heart rate of less than 60 beats/min hence the conflicting results ${ }^{17}$.

Our study protocol was to discontinue the infusion when baroreceptor mediated bradycardia occurred associated with well-maintained blood pressure, rather than the administration of an anticholinergic: hence the number of patients who received atropine was much lower than those who developed bradycardia. According to this work, the best way to reduce hypotension and avoid reactive hypertension and bradycardia is by using a prophylactic phenylephrine infusion at a dose adjusted according to the patient's weight, with supplemental boluses administered as necessary.
There was no statistically significant difference between the two groups in the amount of intravenous fluid Ringers lactate used Table 5. Similarly in this study there was no difference in the number of physician interventions between the control and the intervention arm in the entire duration of the study. According to the study protocol if the blood pressure dropped below $20 \%$ of the baseline a phenylephrine $50 \mu \mathrm{g}$ bolus was given. As seen in table 5 more patients in the control arm received phenylephrine bolus $17 \%$ versus only $7 \%$ in the intervention group. This is due to the fact that the incidence rate of hypotension was significantly higher in the control group as compared to the intervention group Table 4. Similarly the study protocol was to stop the phenylephrine infusion every time the patients' blood pressure increased above $20 \%$ of the baseline. Although the infusion was stopped more frequently in the control group than in the intervention group this difference was not statistically significant. This study was however not adequately powered to detect these differences in the secondary outcomes.

No patient required conversion to general anaesthesia in this study, and a majority of patients achieved a sensory level of T6 before surgery began.

Although not reported, we did not see any adverse effects on the fetus in our study as indicated by the Apgar score that was part of the data collected. However it is well known that any degree of decrease in maternal cardiac output or blood pressure may have detrimental effect on the fetus: by causing a reduction in placental blood flow and subsequently a reduction in oxygen delivery to the fetus. This is even worse in the emergency situation where fetal acidosis may already be present.

In such situations it is important that the anesthesiologist optimizes oxygen delivery to the fetus by maintaining maternal blood pressure and cardiac output as near normal as possible. Hence based on the findings of this study a prophylactic weight adjusted phenylephrine infusion will result in a lower drop in the maternal systolic blood pressure and also less bradycardia and less reactive hypertension.

\section{Strengths of the study}

After a rigorous literature review, it appears that this is the first prospective randomized controlled study that has 
compared a prophylactic fixed dose infusion to a weight adjusted dose infusion. Therefore, this study will add to the body of literature and knowledge on prevention of hypotension following spinal anesthesia given for caesarean section and probably form a basis for many other studies in the future.

\section{Limitations of the study}

The study was conducted at a single centre and involved a relatively small number of patients; this may impact on the generalizability of the results of this study.

The calculation of the power of the current study was based on a small number of anecdotal case series, and not a large randomized clinical trial due to paucity of published studies on prophylactic phenylephrine weight adjusted dose infusion. The case series was retrospective, no protocol was involved in the administration of fluids and vasopressors and the analysis was not rigorous enough. This probably explains discrepancy between the postulated proportion of hypotension and the findings of the study

\section{Conclusion}

On the basis of the results of this study, patients in the weight adjusted dose group had a lower incidence of hypotension compared to those in the fixed dose group. This difference was of borderline statistical significance. The difference in heart rate change (bradycardia) between the two groups and reactive hypertension was not statistically significant. Therefore, we conclude that weight adjusting the dose of prophylactic phenylephrine infusion at $0.5 \mu \mathrm{g} / \mathrm{kg} / \mathrm{min}$ results in a lower incidence of hypotension, slightly lower incidence of reactive hypertension and bradycardia than administering it at a fixed dose of $37.5 \mu \mathrm{g} / \mathrm{min}$ to all patients, regardless of their weight.

\section{References}

1. Tagaloa LA, Butwick AJ, Carvalho B. A Survey of Perioperative and Postoperative Anesthetic Practices for Cesarean delivery. Anesthesiol Resp Pract 2009: http://dx.doi. org/10.1155/2009/510642

2. Reynolds F, Seed PT. Anaesthesia for Caesarean section and neonatal acid-base status: a meta-analysis. Anaesthesia. 2005; 60(7):636-53.http://onlinelibrary.wiley. com/doi/10.1111/j.1365-2044.2005.04223.x/pdf

3. Quin a C, Milne D, Colum M, GortonH, Knight M. Failed tracheal intubation in obstetric anaesthesia: 2 yr na- tional case-control study in the UK. Br J Anaesth. 2013:7480. http://dx.doi.org/10.1093/bja/aes320

4. Hawkins JL. Anesthesia-Related Maternal Mortality. Clin Obstet Gynecol 2003; 46 (3) 679-87. http://dx.doi. org/10.1097/00003081-200309000-00020

5. Musaid RA, Naranjo TM. Spinal block complications in obstetrics. Neurosciences Riyadh 2006; 113:140-4

6. Hartmann B, Junger A, Klasen J, Benson M, Jost A, Banzhaf $A$ et al. The incidence and risk factors for hypotension after spinal anesthesia induction: an analysis with automated data collection. Anesth Analg. 2002:1521-9. http://dx.doi.org/10.1213/00000539-200206000-00027 7. Mitra JK. Prevention of hypotension following spinal anaesthesia in caesarean section -then and now. Kathmandu Univ Med J. 2010; 415-9

8. Stewart A, Fernando R, McDonald S, Hignett R, Jones T, Columb M. The dose-dependent effects of phenylephrine for elective cesarean delivery under spinal anesthesia. Anesth Analg 2010; 1115: 1230-7. http://dx.doi. org/10.1213/ANE.0b013e3181f2eae1

9. Cyna AM, Andrew M, Emmett RS, Middleton P, Simmons SW. Techniques for preventing hypotension during spinal anaesthesia for caesarean section. Cochrane Database Syst Rev 2006; 184 :CD 002251

10. Warwick D, Ngan Kee et al. Prevention of hypotension during spinal anesthesia for cesarean section. Anesthesiology 2005; 4:744-50

11. Banerjee A, Stocche RM. Preload or coload for spinal anesthesia for elective Cesarean delivery: a metaanalysis ' Can J of Anaesth. 2010; 24-31 .http://dx.doi. org/10.1007/s12630-009-9206-7

12. Mercier Fredric. Fluid Loading for Cesarean Delivery Under Spinal Anesthesia. Anesth Analg 2011;1134:677 -80. http://dx.doi.org/10.1213/ANE.0b013e3182245af4

13. Rout CC, Rocke DA, Levin J, Gouws E, Reddy D: A Re-evaluation of the Role of Crystalloid Preload for the prevention of hypotension during spinal anesthesia for caesarean section. Anesthesiology 1993: 79 2; 262-269.

14. Lee A, Ngan Kee WD, Gin T. A quantitative, systematic review of randomized controlled trials of ephedrine versus phenylephrine for the management of hypotension during spinal anesthesia for cesarean delivery. Anesth Analg. 2002 Apr; 944:920-6. http://dx.doi. org/10.1097/00000539-200204000-00028

15. Ngan Kee WD, Lee A, Khaw KS, Ng FF, Karmakar MK, Gin T. A randomized double-blinded comparison of phenylephrine and ephedrine infusion combinations 
to maintain blood pressure during spinal anesthesia for cesarean delivery: the effects on fetal acid-base status and hemodynamic control. Anesth Analg. 2008; 107 4:1295302. http://dx.doi.org/10.1213/ane.0b013e31818065bc 16. Ngan Kee WD, Khaw KS, Ng FF, Lee BB. Prophylactic Phenylephrine Infusion for Preventing Hypotension during Spinal Anesthesia for Cesarean Delivery. Anesth Analg. 2004; 98 3:815-21. http://dx.doi.org/10.1213/01. ANE.0000099782.78002.30

17. Allen TK, George RB, White WD, Muir H a, Habib AS. A double-blind, placebo-controlled trial of four fixed rate infusion regimens of phenylephrine for hemodynamic support during spinal anesthesia for cesarean delivery. Anesth Analg. 2010; 1115:1221-9. http://dx.doi. org/10.1213/ANE.0b013e3181e1db21

18. Habib AS. A review of the impact of phenylephrine administration on maternal hemodynamics and maternal and neonatal outcomes in women undergoing cesarean delivery under spinal anesthesia. Anesth Analg. 2012; 1142:377-90. http://dx.doi.org/10.1213/ ANE.0b013e3182373a3e
19. Dawes M, Chowienczyk PJ. Pharmacokinetics in pregnancy. Best practice \& Research Clin Obstet Gynecol 2001; 156:819-26.http://dx.doi.org/10.1053/beog.2001.0231 20. Pai MP. Drug dosing based on weight and body surface area: mathematical assumptions and limitations in obese adults. Pharmacotherapy. 2012 Sep; 329:856-68. http://dx.doi.org/10.1002/j.1875-9114.2012.01108.x

21. Schering Plough Corporation Summi, NJ. Understanding Phenylephrine Metabolism, Pharmacokinetics, Bioavailability and Activity. Briefing document for NDAC meeting 2007; 1-9.

22. Pharmd JTM, Pharmd LSW, Pharmd MDK, Alaniz C. Does body weight impact the efficacy of vasopressin therapy in the management of septic shock? J of Crit Care; 2012; 273:289-93. http://dx.doi.org/10.1016/j. jcrc.2011.06.018

23. Dyer RA, Reed AR. Spinal hypotension during elective cesarean delivery: closer to a solution. Anesth Analg. 2010; 1115:1093-5.http://doi.org/10.1213/ ANE.0b013e3181ea5f77 